\title{
BMJ Open Dexamethasone versus prednisone for children receiving asthma treatment in the paediatric inpatient population: protocol for a feasibility randomised controlled trial
}

Catherine M Pound, ${ }^{1}$ Jaime McDonald, ${ }^{2}$ Ken Tang, ${ }^{3}$ Gillian Seidman, ${ }^{1}$ Radha Jetty, ${ }^{1}$ Sarah Zaidi, ${ }^{3}$ Amy C Plint ${ }^{4}$

To cite: Pound CM, McDonald J, Tang K, et al. Dexamethasone versus prednisone for children receiving asthma treatment in the paediatric inpatient population: protocol for a feasibility randomised controlled trial. BMJ Open 2018;8:e025630. doi:10.1136/ bmjopen-2018-025630

- Prepublication history and additional material for this paper are available online. To view these files, please visit the journal online (http://dx.doi. org/10.1136/bmjopen-2018025630).

Received 25 July 2018 Revised 9 0ctober 2018 Accepted 19 October 2018

Check for updates

(C) Author(s) (or their employer(s)) 2018. Re-use permitted under CC BY-NC. No commercial re-use. See rights and permissions. Published by BMJ.

For numbered affiliations see end of article.

Correspondence to Dr Catherine M Pound; cpound@cheo.on.ca

\section{ABSTRACT}

Introduction Asthma exacerbations are a leading cause of paediatric hospitalisations. Corticosteroids are key in the treatment of asthma exacerbations. Most current corticosteroids treatment regimens for children admitted with asthma exacerbation consist of a 5-day course of prednisone or prednisolone. However, these medications are associated with poor taste and significant vomiting, resulting in poor compliance with the treatment course. While some centres already use a short course of dexamethasone for treating children hospitalised with asthma, there is no evidence to support this practice in the inpatient population.

Methods and analysis This single-site, pragmatic, feasibility randomised controlled trial will determine the feasibility of a non-inferiority trial, comparing two treatment regimens for children admitted to the hospital and receiving asthma treatment. Children 18 months to 17 years presenting to a Canadian tertiary care centre will be randomised to receive either a short course of dexamethasone or a longer course of prednisone/ prednisolone once admitted to the inpatient units. The primary clinical outcome for this feasibility study will be readmission to hospital or repeat emergency department visits, or unplanned visits to primary healthcare providers for asthma symptoms within 4 weeks of hospital discharge. Feasibility outcomes will include recruitment and allocation success, compliance with study procedures, retention rate, and safety and tolerability of study medications. We plan on recruiting 51 children, and between-group comparisons of the clinical outcome will be conducted to gain insights on probable effect sizes.

Ethics and dissemination Research Ethics Board approval has been obtained for this study. The results of this study will inform a multisite trial comparing prednisone/prednisolone to dexamethasone in inpatient asthma treatment, which will have the potential to improve the delivery of asthma care, by improving compliance with a mainstay of treatment. Results will be disseminated through peer-reviewed publications, organisations and meetings.

Trial registration number NCT03133897; Pre-results.
Strengths and limitation of this study

- This pragmatic feasibility trial paves the way for a large multisite pragmatic trial which will compare a short course of dexamethasone to a longer course of prednisone/prednisolone in the treatment of patients admitted to the hospital with asthma symptoms.

- As patients are enrolled after the decision to admit to hospital has been made, there is no restriction on the type of corticosteroid prescribed by the emergency department physician on presentation, allowing for generalisability and applicability to most paediatric clinical settings.

- Participants and healthcare providers will not be blinded to the intervention; however, this will allow for better generalisability and interpretation of results as palatability of prescribed corticosteroids and length of treatment are known to affect compliance, which would both be lost with blinding.

\section{INTRODUCTION}

Asthma is the most common chronic disease of childhood, ${ }^{1}$ affecting approximately $15 \%$ of children and youth in Canada. ${ }^{2}$ Asthma continues to be one of the leading causes of hospitalisation in children. ${ }^{3}$ In Ontario, the burden of illness related to asthma is enormous, accounting for one-third of government health expenditures for the general population. ${ }^{4}$

A key element for treating children presenting with symptoms consistent with acute asthma exacerbations is the administration of systemic corticosteroids (CSs). Systemic CSs reduce the need for hospitalisation and the risk of relapse after initial treatment, and may also facilitate an earlier discharge from the hospital. ${ }^{5}$ Some centres already use a short course of dexamethasone for inpatient asthma management. However, 
while data are promising for outpatients, there is no evidence to support this practice in the inpatient population. Most current CS regimens for the treatment of children hospitalised with an asthma exacerbation consist of a 5-day course of prednisone or prednisolone.

There is evidence that 2 days of once daily dexamethasone is a least as effective as 5 days of prednisone for preventing relapse in outpatient paediatric asthma. ${ }^{6}$ In addition, patients receiving dexamethasone are significantly less likely to experience vomiting in the emergency department (ED) and even after returning home. ${ }^{6}$ Preliminary cost estimates suggest that dexamethasone may save at least US $\$ 3500$ per 100 patients compared with traditional treatment with prednisone or prednisolone. ${ }^{7}$

Prednisone is only available in Canada as a tablet or compounded suspension, which limits use due to swallowing ability and accessibility. When compared with dexamethasone, prednisolone is associated with poor palatability and significant vomiting. ${ }^{8}$ Both prednisone and prednisolone are associated with poor compliance. Prednisone and prednisolone are compounded with similar recipes, and therefore have a similar taste. Compliance with 5 days of prednisone has been estimated in one study to be as low as $64 \%$ for paediatric asthma. ${ }^{9} \mathrm{As}$ determined by a systematic review conducted by the principal investigator (PI) of this current study (PROSPERO 2016:CRD42016041766), studies comparing dexamethasone to prednisone in hospitalised patients are lacking. Promisingly, a retrospective cohort study in paediatric inpatients hospitalised with asthma suggested that dexamethasone, when compared with prednisone/prednisolone, may result in a shorter length of hospital stay and reduced costs with no difference in number of transfers to intensive care or readmissions. ${ }^{10}$

Dexamethasone is a potent glucocorticoid with a long half-life, therefore, concerns have been raised regarding its potential for adrenal suppression. However, a study of high-dose $(\sim 1.7 \mathrm{mg} / \mathrm{kg})$ dexamethasone in acute asthma found no significant difference in adrenal function between single-dose dexamethasone and 5 days of oral prednisone at day $14 .{ }^{11}$

Given the importance of CS in the treatment of asthma, the significant decrease in relapse risk associated with their use and the high prevalence of childhood asthma, there is a need to determine whether a better tolerated and more convenient CS can be used as first-line therapy. Although outpatient data on this issue exist, extrapolating treatment regimens from the outpatient population may not be appropriate as hospitalised children represent a sicker group of patients than those discharged from the ED.

In order to determine whether dexamethasone is at least as effective as prednisone/prednisolone in the treatment of inpatient asthma, we propose a feasibility study, as a first step in the development of a future multisite trial.

Specifically, we plan to determine the feasibility of a non-inferiority trial, comparing a short course of dexamethasone to the more traditional longer course of prednisone/prednisolone for children admitted to the hospital and receiving asthma treatment. We will determine the feasibility of:

1. Enrolling patients on admission to hospital, after they receive their first dose(s) of CS in the ED.

2. Asking patients and/or caregivers to complete a symptom diary weekly for 4 weeks.

3. Reassessing patients 7 days after hospital admission day.

4. Successfully completing phone follow-up 4 weeks posthospital discharge.

5. Collecting health utilisation data posthospital discharge.

\section{METHODS AND ANALYSIS}

\section{Trial design}

This trial will be conducted at the Children's Hospital of Eastern Ontario (CHEO) in Ottawa, Ontario, Canada. Children with asthma, admitted from the ED to the clinical teaching units, will be eligible for the study (see figure 1). Trial recruitment commenced in February 2018. This protocol follows Standard Protocol Items: Recommendations for Interventional Trials guidelines figure 1 .

A pragmatic design ${ }^{12}$ was chosen to strengthen the generalisability of our findings, as the effectiveness of the intervention is being tested across routine clinical practice. Blinding of the participants and members of the healthcare team will not be undertaken in this study. Since the palatability of the CS is likely to affect compliance, making the taste of the CS similar to ensure blinding would decrease ability to detect a difference in compliance due to taste. Moreover, given the length of therapy for prednisone/prednisolone is longer than that of dexamethasone, introducing placebo doses to ensure similar length of treatment would also potentially impact compliance and undermine the ability to detect a difference between groups. Investigators, data analysts and research assistant completing patient assessment at the follow-up visit will however be blinded to group assignment.

\section{Eligibility criteria}

\section{Inclusion criteria}

Participants must meet all of the inclusion criteria listed below to participate in the study:

- Children admitted during the study period who are receiving asthma treatment for acute respiratory exacerbation as determined by the admitting physician and/or his/her representative. Of note, given the pragmatic design of the study, and that clinicians cannot always establish a diagnosis of asthma with certainty when a child presents to the hospital, children receiving asthma treatment will be considered for this study.

- Children aged 18 months to 17 years of age.

- Children who have received oral or intravenous CS in the ED prior to admission under the institution's 


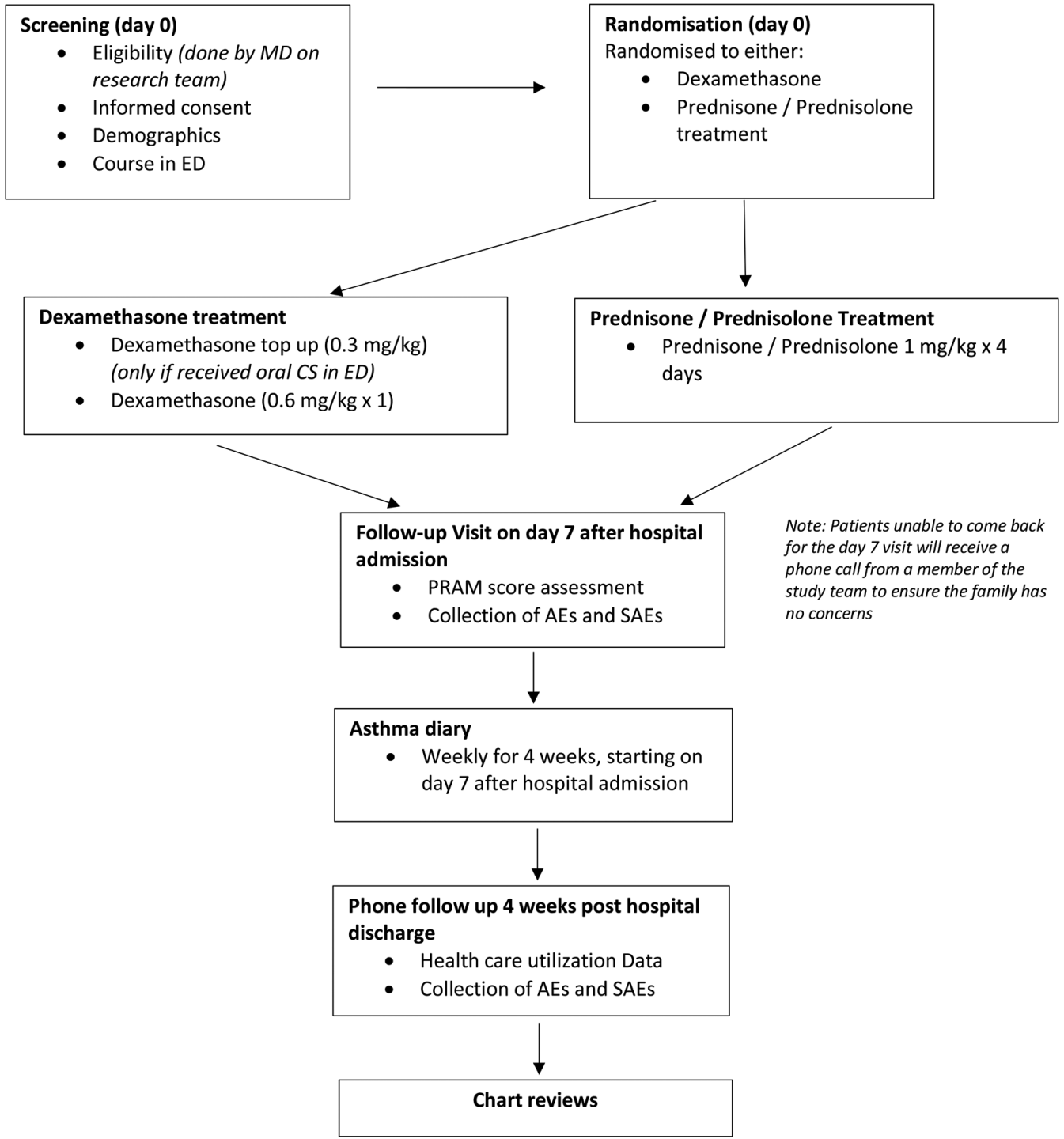

Figure 1 Schematic of study design. AEs, adverse events; CS, corticosteroid; ED, emergency department; PRAM, Paediatric Respiratory Assessment Measure; SAEs, serious adverse events.

'Physician Orders for Asthma in the ED' and 'Physician Orders for Asthma Respiratory Failure in the ED' order sets (POS) or 'Emergency Department Nursing Medical Directive' (see online supplementary files $1-3$, respectively).

- Children who have received intravenous CS for a total duration $\leq 24$ hours since first dose of steroid administration in ED.

- Participants must be capable of giving informed consent or have an acceptable surrogate capable of giving consent on their behalf.

\section{Exclusion criteria}

All candidates meeting any of the exclusion criteria at baseline will be excluded from study participation.

- Children who received oral or intravenous CS in the ED prescribed in any other way than the institution's POS or Nursing Medical Directive as doses may not be standardised.
- Children who have received more than one dose of oral CS prior to enrolment.

- Children who received oral or intravenous CS in the previous 2 weeks.

- Children with any of the following: unrepaired congenital heart disease, cardiac disorder, chronic lung diseases other than asthma, such as bronchopulmonary dysplasia or cystic fibrosis, neurological or neuromuscular disease, sickle cell disease.

- Children presenting with stridor.

- Children admitted to the paediatric intensive care unit.

- Children whose caregivers do not understand English or French.

\section{Intervention}

Study medication description: dexamethasone

Children randomised to the dexamethasone group will receive the approximate pharmacological equivalent of two 
doses (days) of dexamethasone $0.6 \mathrm{mg} / \mathrm{kg} / \mathrm{dose}$ (maximum dose $16 \mathrm{mg}$ per dose) as current evidence supports the use of this dose for the inpatient population. ${ }^{10} 13$

The standard dose of dexamethasone provided in the $\mathrm{ED}$ is $0.3 \mathrm{mg} / \mathrm{kg}$, while the standard dose of prednisone/ prednisolone is $2 \mathrm{mg} / \mathrm{kg}$ (maximum single dose $50 \mathrm{mg}$ ), which is approximately equivalent to $0.3 \mathrm{mg} / \mathrm{kg}$ of dexamethasone. ${ }^{14}$ Once enrolled, children who received dexamethasone $0.3 \mathrm{mg} / \mathrm{kg}$ or prednisone $2 \mathrm{mg} / \mathrm{kg}$ in the ED will be administered a 'top-up' dose of dexamethasone at $0.3 \mathrm{mg} / \mathrm{kg}$ (maximum dose $8 \mathrm{mg}$ ). They will then receive a dose of dexamethasone $0.6 \mathrm{mg} / \mathrm{kg}$ (maximum dose $16 \mathrm{mg}) 24 \pm 6$ hours after the initial CS dose received in the ED (see online supplementary file 4 for study order sheet). The dexamethasone dose will be rounded to the nearest $0.25 \mathrm{mg}$ on initial prescribing. Deviations of up to $10 \%$ of the recommended $\mathrm{mg}$ dose per kilogram will be permitted for the purposes of the pharmacist rounding to available dosage forms.

Children who have received intravenous methylprednisolone in the ED and who are randomised to the dexamethasone group will not receive a top-up dose on study enrolment. They will receive one dose (day) of dexamethasone $0.6 \mathrm{mg} /$ $\mathrm{kg} /$ dose (maximum dose $16 \mathrm{mg}$ per dose) 24 hours after the initial dose of oral CS in the ED and at least 6 hours after the last dose of intravenous methylprednisolone (see online supplementary file 4 for study order sheet).

\section{Standard of care description: prednisone/prednisolone}

Children randomised to the prednisone/prednisolone group will receive four doses (days) of prednisone/ prednisolone $1 \mathrm{mg} / \mathrm{kg}$ / dose once daily (maximum single dose $50 \mathrm{mg}$ ) following the initial dose of CS received in the ED (see online supplementary file 4 for study order sheet). The prednisone dose will be rounded to the nearest $0.5 \mathrm{mg}$ on initial prescribing. Deviations of up to $10 \%$ of the recommended mg dose per kilogram will be permitted for the purposes of the pharmacist rounding to available dosage forms.

Children who have received intravenous methylprednisolone in the ED and are randomised to the prednisone/prednisolonegroup will receive four doses (days) of prednisone/prednisolone $1 \mathrm{mg} / \mathrm{kg} /$ dose daily (maximum dose $50 \mathrm{mg}$ ). Study medication will be given 24 hours after the initial dose of oral CS in the ED and at least 6 hours after the last dose of intravenous methylprednisolone (see online supplementary file 4 for study order sheet).

Children in both groups will receive asthma standard of care as dictated by their treating healthcare team, in addition to the study drug.

\section{Study outcomes}

Primary clinical outcome for feasibility study

(1) Readmission to hospital for asthma symptoms, repeat ED visit within 4 weeks for asthma symptoms or unplanned visits to primary healthcare providers for asthma symptoms.
Feasibility outcomes

1. Allocation success; proportion receiving assigned CS as per randomisation group.

2. Recruitment success; number screened, number eligible, number enrolled.

3. Compliance with reporting symptoms in diary.

4. Retention rate: (A) Proportion of patients coming back for follow-up visits; (B) Proportion of patients reached at 4 week follow-up.

5. Safety of each CS.

6. Tolerability of each CS.

\section{Clinical outcomes for multicentre study}

The primary outcome for the multicentre study will be finalised following the feasibility study. As such, clinically relevant outcomes that will be measured in this feasibility study include:

1. Readmission to hospital for asthma symptoms 4 weeks of ED presentation determined using parent report and CHEO chart review.

2. Repeat ED visits for asthma symptoms within 4 weeks of ED presentation determined using parent report and CHEO chart review.

3. Unplanned visits to physicians/nurse practitioners for asthma symptoms measured by parent report and CHEO chart review.

4. Paediatric Respiratory Assessment Measure (PRAM) as measured on day 7 by a research nurse or study team physician. The PRAM score is a validated and reliable tool used to determine asthma severity in children. ${ }^{15}$ Using PRAM score, rather than symptom recurrence, is more precise as a measure of symptom severity and has been used in a recent outpatient paediatric asthma randomised controlled trial comparing dexamethasone and prednisolone. ${ }^{16}$ Health utilisation data (readmissions, ED visits, unplanned visits to family physicians) will be determined through phone recall and health records.

5. Number of children completing assigned CS treatment.

6. Asthma symptom frequency as measured by diary.

7. Length of hospital stay in hours.

8. Vomiting associated with CS administration as determined by parental interview and medical record review.

\section{Enrolment and screening}

A research assistant will review the list of admissions for the previous 12 hours, on a twice daily basis, from Monday to Friday. The research assistant will then ask a member of the patient's healthcare team (bedside nurse, charge nurse, resident and staff physician) to ask the family members for permission to approach the family about the study. The research assistant will then complete a screening $\log$ and maintain a phone follow-up ledger for consented patients. If the patient is eligible and the family gives their informed consent, and the child their assent if appropriate, they will be allocated to one of the 
study groups. Once written informed consent has been obtained, the research assistant will communicate with the healthcare team to ensure the patient continues on the CS he/she has been randomised to, for the appropriate number of doses as determined by CS group assignment. A record will be kept of all screened patients, patient eligibility, allocation and follow-up to allow reporting according to Consolidated Standards of Reporting Trials guidelines. ${ }^{17}$

\section{Randomisation and baseline visit (hospitalisation)}

After enrolment, the patients will be randomly allocated to one of the two treatment groups (day 0 ). The randomization schedule will have been previously generated using a computer. Randomisation will be blocked with randomly chosen block lengths of 4 or 6 . Treatment assignments will be written on a piece of paper and concealed in sequentially numbered opaque envelopes kept in a secure locked location in the study research office. The PI and analyst will be blinded to the treatment intervention, but the research assistant in charge of screening and randomising patients, as well as the patient's treating team (physicians and nurses) will not because of the pragmatic nature of the trial. Demographic data will be collected at baseline.

\section{Follow-up visit}

Patients will be reassessed 7 days after admission day to hospital, \pm 3 days to allow for flexibility with patient's schedule, weekends, holidays and unexpected events. Patients will receive a physical examination by a study physician, and an assessment of asthma symptom severity through the PRAM score. ${ }^{15}$ At that visit, we will collect compliance data with both courses of treatment, and remind participants to begin charting asthma symptoms in their symptom diary. PRAM scores at the follow-up visit will be collected on the case report form. Data on adverse events (AEs) and serious AEs (SAE) will also be collected at that visit. Patients unable to come back for the day 7 visit will receive a phone call from a study physician to ensure the family has no concerns, and compliance data with course of treatment will be collected via phone. If the participant consents, a partial PRAM assessment will be done via video conference call by the study physician.

\section{Phone follow-up}

Phone follow-up will be performed at 4 weeks posthospital discharge, \pm 7 days to allow for flexibility with patient's schedule, weekends, holidays and unexpected events. Data on return visits to healthcare providers, ED visits and hospital readmissions will be collected by phone 4 weeks posthospital discharge, as well as data on AE and SAE. We will also collect compliance data with both courses of treatment if the information could not be obtained earlier.

Data on patients' experience with prescribed CS will also be collected at the follow-up phone call via semi-structured phone interviews.

\section{Chart review}

At the end of each subject's participation period, the research assistant will review the medical chart and extract information on treatment course in the ED and on the inpatient ward, including date, time and dosage of CS and antibiotics administered. Data on length of stay will also be obtained. Charts will also be reviewed for subsequent visits to the ED, and for admissions for asthma symptoms in the 4 weeks following hospital discharge.

\section{Expected duration of participant participation}

Study subjects are expected to participate in the study from enrolment until 4 weeks after hospital discharge (see figure 1).

Parents and/or caregivers in both study groups will be asked to electronically complete a standardised validated symptom diary weekly, starting 7 days postdischarge until 4 weeks post discharge (The Asthma Quiz for Kidz). Patients who prefer a paper copy of the questionnaire will be provided with four questionnaires prior to hospital discharge, as well as preaddressed stamped envelopes to facilitate mailing back of the questionnaire. The Asthma Quiz for Kidz is a short 6-item questionnaire validated for in French and English for children aged 1-17years.

\section{Formulation, packaging and labelling Dexamethasone}

Patients will receive dexamethasone orally as tablet(s) or compounded suspension based on patient or healthcare provider preference. The dexamethasone suspension will be compounded at the CHEO research pharmacy using a combination of dexamethasone sodium phosphate USP injection $10 \mathrm{mg} / \mathrm{mL}$ ( ${ }^{\mathrm{PR}}$ Dexamethasone Omega Unidose; see product monograph) manufactured by Omega Laboratories. and Ora-Blend, a flavoured oral suspending vehicle manufactured by Galenova. The compound is created by combining $10 \mathrm{~mL}$ of the dexamethasone $10 \mathrm{mg} / \mathrm{mL}$ sodium phosphate USP and $90 \mathrm{~mL}$ of Ora-Blend. The final oral suspension has a concentration of $1 \mathrm{mg} / \mathrm{mL}$ of dexamethasone and a pinkish hue with a milky consistency. The appropriate dose for the participant will be drawn up into an oral syringe, which will be labelled for investigational use.

\section{Prednisone/prednisolone}

Patients will receive prednisone/prednisolone orally as tablet(s) or compounded suspension (prednisone) or as a commercially available liquid (prednisolone). The prednisone suspension will be compounded at the CHEO research pharmacy using a combination of APO-prednisone $50 \mathrm{mg}$ tablets ( ${ }^{\mathrm{PR}} \mathrm{APO}-\mathrm{PREDNISONE} 50 \mathrm{mg}$ tablet; see product monograph) and Syrup, NF (Simple) a sweetened vehicle manufactured by Medisca at a ratio of one $50 \mathrm{mg}$ tablet for every $10 \mathrm{~mL}$ of Syrup, NF (Simple). The final oral liquid has a concentration of $5 \mathrm{mg} / \mathrm{mL}$ with a yellow to white hue and clear to milky consistency. The appropriate dose for the participant will be drawn up into an oral syringe, which will be labelled for investigational use. 


\section{Sample size determination}

We sought a sample size that would allow us to estimate our feasibility outcomes with reasonable precision. As such, we have set out to achieve a $15 \%$ margin of error (ie, half-width of $95 \% \mathrm{CI}=0.15$ ) for any proportions to be estimated (eg, allocation success, retention success). At a hypothesised proportion of $50 \%$, we determined that 43 patients will be needed to achieve this level of precision. This provides a most conservative estimate since any proportions other than $50 \%$ will require fewer patients. Factoring in an additional $15 \%$ of patients as allowance for attrition or incomplete data, we will set a recruitment target of $n=51$ in total. With approximately 18 asthma admissions per month, assuming $70 \%$ are eligible and $50 \%$ of eligible patients consent, we expect to meet this sample size requirement in 9 months.

\section{Quantitative data analysis}

For all relevant feasibility outcomes, binary proportions (eg, \% success) and the associated $95 \%$ CI will be estimated using the Wilson method. To help inform the design of main trial (ie, expected effect sizes), we will apply intention-to-treat principles and estimate the treatment effect (and 95\% CI) by comparing outcomes (proposed for the main trial) between intervention groups using statistical techniques appropriate to the type and distribution of the various outcomes (eg, continuous, binary, count data).

\section{Qualitative data analysis}

Semistructured interviews at week 4 will be audio taped and transcribed verbatim. Analyses will be conducted using NVivo V.9 software. Inductive analysis will be used to identify categories, patterns and themes. ${ }^{18}$

\section{Role of the data safety monitoring board}

A data safety monitoring board (DSMB) will be set up for this study. An initial meeting will occur prior to enrolling the first patient to determine the terms of reference, review Health Canada-mandated SAE reporting and safety outcomes. Given that this trial is a feasibility trial as opposed to an efficacy trial, no interim analysis for efficacy will be performed. The DSMB will meet every 4 months after the initial meeting, until study completion. Study results will be analysed after all participants have completed the study.

\section{SAFETY AND AES}

Patient safety will be ensured by using standard paediatric dosing, ${ }^{10} 13$ by adhering to the inclusion and exclusion criteria, and by following the current standard of care for mitigation of treatment side effects. Patients whose PRAM scores are greater than 1 on follow-up visits will be treated appropriately. A follow-up with a study physician will be booked if necessary. The patient's family physician will also be contacted to ensure appropriate follow-up. The patient's family will be instructed to come to the ED immediately in the case of symptom worsening, and to page the study physician who assessed the patient. Patients whose PRAM scores are greater than 3 on follow-up visits will be immediately sent to the CHEO ED.

Patients unable to come back for the day 7 visit will receive a phone call from a member of the study team to ensure the family has no concerns. If health concerns are identified, patients will be asked to come to CHEO to be assessed by a member of the study team. If the family refuses, patients will be encouraged to visit the closest ED or to visit their family physician. A member of the investigative team will also call the family physician's office to ensure the patient is seen promptly.

\section{MONITORING}

The investigator will ensure the trial is adequately monitored in accordance to the protocol and applicable regulatory requirements. An internal monitoring visit will be completed by the CHEO Research Institute Quality Assurance (QA) team after the first participant is enrolled and further monitoring by the QA team can be requested by the investigator, if required. Peer review, on-site monitoring visits will be completed by an investigator-appointed monitor who is familiar with the study medication and protocol. The monitor will ensure that the trial is conducted and documented according to Good Clinical Practices (GCPs). As a Health Canada regulated trial, this study may be inspected at any time by Health Canada.

\section{DATA HANDLING AND RECORD KEEPING \\ Data management responsibilities}

Information will be collected for each participant by a member of the research study team at each point of study data collection. Participants will be assigned a randomisation code at baseline and their records will be identified using this code so that all study subjects are non-identifiable by their study documents.

The PI and research assistant will be responsible for the overall supervision of the study. The research assistant will be responsible for recruiting patients, communicating with the nursing staff to ensure patients receive the right CS after the initial dose received in the ED, supervising the chart review and conducting the phone follow ups. Study team physicians will be responsible for patient reassessment 7 days after initial presentation.

Subject data will be recorded on source documents and transferred to Research Electronic Data Capture (REDCap). REDCap is a secure, web-based application designed exclusively to support data capture for research studies. REDCap provides: (1) an intuitive interface for data entry (with data validation); (2) 128 bit encryption between the data entry client and the server (https); (3) audit trails for tracking data manipulation and export procedures; (4) automated export procedures for seamless data downloads to $R$ version 3.3.2 (REF); (5) procedures for importing data from external sources and (6) 
advanced features, such as branching logic and calculated fields.

REDCap is developed and maintained by a team at Vanderbilt University and licensed free of charge by the Research Institute at CHEO. The application and data are housed on servers provided by CHEO. These servers are located within CHEO's secure data centre. The data centre is physically secured through limited badge access and security cameras. Local support for REDCap is provided by CHEO's Clinical Research Unit.

\section{Confidentiality}

All participant-related information including case report forms, laboratory specimens, reports will be kept strictly confidential. All records will be kept in a secure, locked location and only research staff will have access to the records. Participants will be identified only by means of a coded number specific to each participant. All computerised databases will identify participants by numeric codes only and will be password protected or encrypted.

On request, participant records will be made available to the study sponsor, monitoring groups representative of the study sponsor, representatives of a participating pharmaceutical sponsor and applicable regulatory agencies such as Health Canada or The Food and Drug Agency.

\section{Record retention}

All research records will be retained for 25 years after closure as required by Health Canada. All study documentation will be kept in a secure location at the study site.

\section{Data safety monitoring board}

This study will be monitored by an independent DSMB, consisting of an independent physician and two other members not involved in this study. The DSMB will be immediately informed of any SAE which may potentially be study drug related. Other SAEs will be reviewed during regular DSMB meetings. Interim reports, prepared by the data management team for the study, for review by the DSMB will include data on recruitment, compliance, adverse effects, baseline comparability and treatment comparisons. An agreed on blinded review package which contains the appropriate data summary by treatment will be provided by the study statistician for the purposes of these reviews. The review package could then be unblinded at the request of the DMSB.

\section{Patient and public involvement}

Patients and the public were not directly involved in the design of the study. The intervention was chosen on the basis of studies reporting poor compliance with current CS used in the treatment of inpatient asthma ${ }^{9}$ due to poor palatability of the drug and associated vomiting. ${ }^{8}$ Study results will be disseminated through patients and study participants through our institution's social media platform.

\section{Ethical considerations}

This study will be conducted in full conformance with the principles of the 'Declaration of Helsinki', GCP and within the laws and regulations of Canada (Health Canada, Food and Drug Act, Part C, Division 5, Drugs for Clinical Trials Involving Human Subjects).

The decision of the Research Ethics Board (REB) concerning the conduct of the study will be made in writing to the investigator.

All participants for this study will be provided a consent form and assent form if applicable, describing this study and providing sufficient information for participants to make an informed decision about their participation in this study. These consent and assent forms were submitted with the protocol for review and approval by the REB. The formal consent of a participant, using the REB-approved consent form, will be obtained before that participant is submitted to any study procedure. This consent form must be signed by the participant or legally acceptable surrogate and the investigator-designated research professional obtaining the consent. Patients will be free to withdraw from study participation at any point in time and will receive the same standard of medical care given to all patients admitted with asthma to the paediatric ward at CHEO. The data collected during this trial will be held confidential.

All study investigators will have access to the final trial dataset. The International Committee of Medical Journal Editors authorship eligibility guidelines will be used for publication. We plan on disseminating our study results through peer-reviewed publications, professional organisations and conferences.

\section{Author affiliations}

${ }^{1}$ Department of Pediatrics, University of Ottawa, Children's Hospital of Eastern Ontario, Ottawa, Ontario, Canada

${ }^{2}$ Clinical Pharmacist, Izaak Walton Killam (IWK) Health Centre, Halifax, Nova Scotia, Canada

${ }^{3}$ Clinical Research Unit, Children's Hospital of Eastern Ontario Research Institute, Ottawa, Ontario, Canada

${ }^{4}$ Departments of Pediatrics and Emergency Medicine, Children's Hospital of Eastern Ontario, University of Ottawa, Ottawa, Canada

Acknowledgements The authors wish to thank Danielle Garceau for her invaluable help with implementing the pharmacy procedures. The authors wish to thank Barbara Murchison for her feedback and help in getting the study implemented. The authors wish to thank Sheila Ledoux and Sabrina Hamer for their help with quality assurance and help with protocol submission to Health Canada.

Contributors CMP conceived and designed the study and drafted the first version of the manuscript, and approved the final version of the manuscript. JM, KT, GS and RJ participated in the design of the study, read and reviewed the manuscript and approved the final version of the manuscript. SZ participated in revising the study protocol, read and reviewed the manuscript and approved the final version of the manuscript. ACP participated in and supervised the design of the study, read and reviewed the manuscript and approved the final version of the manuscript.

Funding This work was supported by the Academic Health Sciences Center Alternate Funding Plan Innovation Fund Grant.

Disclaimer The funders had no role in study design, data collection and decision to submit this manuscript for publication, and will have no authority over management, analysis and interpretation of data.

Competing interests None declared. 
Patient consent Not required.

Ethics approval Protocol and amendments will be submitted to the CHEO REB for formal approval to conduct the study. The decision of the Research Ethics Board (REB)concerning the conduct of the study will be made in writing to the investigator.

Provenance and peer review Not commissioned; externally peer reviewed.

Open access This is an open access article distributed in accordance with the Creative Commons Attribution Non Commercial (CC BY-NC 4.0) license, which permits others to distribute, remix, adapt, build upon this work non-commercially, and license their derivative works on different terms, provided the original work is properly cited, appropriate credit is given, any changes made indicated, and the use is non-commercial. See: http://creativecommons.org/licenses/by-nc/4.0/.

\section{REFERENCES}

1. Kovesi T, Schuh S, Spier S, et al. Achieving control of asthma in preschoolers. CMAJ 2010;182:E172-E183.

2. Centre for Surveillance and Applied Research, Public Health Agency of Canada. Canadian Chronic Disease Indicators, 2017 Edition. Ottawa 2017

3. Canadian Institute for Health Information. Asthma Hospitalizations Among Children and Youth in Canada: Trends and Inequalities. Ottawa, ON: CIHI, 2018.

4. To T, Dell S, Dick P, et al. Burden of childhood asthma. Toronto, Ontario: ICES, 2004.

5. Smith M, Iqbal S, Elliott TM, et al. Corticosteroids for hospitalised children with acute asthma. Cochrane Database Syst Rev 2003;2:CD002886

6. Keeney GE, Gray MP, Morrison AK, et al. Dexamethasone for acute asthma exacerbations in children: a meta-analysis. Pediatrics 2014;133:493-9.

7. Andrews AL, Wong KA, Heine D, et al. A cost-effectiveness analysis of dexamethasone versus prednisone in pediatric acute asthma exacerbations. Acad Emerg Med 2012;19:943-8.
8. Hames $\mathrm{H}$, Seabrook JA, Matsui $\mathrm{D}$, et al. A palatability study of a flavored dexamethasone preparation versus prednisolone liquid in children. Can J Clin Pharmacol 2008;15:e95-e98.

9. Butler K, Cooper WO. Adherence of pediatric asthma patients with oral corticosteroid prescriptions following pediatric emergency department visit or hospitalization. Pediatr Emerg Care 2004;20:730-5.

10. Parikh K, Hall M, Mittal V, et al. Comparative effectiveness of dexamethasone versus prednisone in children hospitalized with asthma. J Pediatr 2015;167:639-44.

11. Gries DM, Moffitt DR, Pulos E, et al. A single dose of intramuscularly administered dexamethasone acetate is as effective as oral prednisone to treat asthma exacerbations in young children. $J$ Pediatr 2000;136:298-303.

12. Patsopoulos NA. A pragmatic view on pragmatic trials. Dialogues Clin Neurosci 2011;13:217-24.

13. Meyer JS, Riese J, Biondi E. Is dexamethasone an effective alternative to oral prednisone in the treatment of pediatric asthma exacerbations? Hosp Pediatr 2014;4:172-80.

14. Canadian Pharmaceutical Association Compendium of Pharmaceuticals and Specialties. Toronto: Canadian Pharmaceutical Association, 2013.

15. Ducharme FM, Chalut $D$, Plotnick $L$, et al. The pediatric respiratory assessment measure: A valid clinical score for assessing acute asthma severity from toddlers to teenagers. J Pediatr 2008;152:476-80.

16. Cronin JJ, McCoy S, Kennedy U, et al. A randomized trial of singledose oral dexamethasone versus multidose prednisolone for acute exacerbations of asthma in children who attend the emergency department. Ann Emerg Med 2016;67:593-601.

17. Moher D, Schulz KF, Altman DG. The CONSORT statement: revised recommendations for improving the quality of reports of parallelgroup randomised trials. Lancet 2001;357:1191-4.

18. Patton MQ. Qualitative evaluation and research methods. Los Angeles, CA: Sage, 2002. 